\title{
Application of Fluorescent Dyes in Visceral Surgery: State of the Art and Future Perspectives
}

\author{
Kai Nowak ${ }^{\mathrm{a}}$ Ioannis Karampinis ${ }^{\mathrm{b}}$ Andreas Lutz Heinrich Gerken ${ }^{\mathrm{b}}$

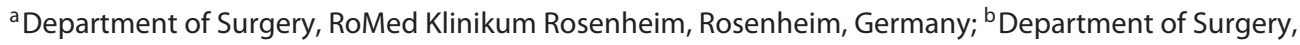 \\ Universitätsmedizin Mannheim, Medical Faculty Mannheim, Mannheim, Germany
}

\section{Keywords}

Fluorescence angiography · Fluorescence imaging · General surgery · Indocyanine green · Intraoperative imaging

\begin{abstract}
Background: Through the improvement and implementation of advanced intraoperative imaging, the indications for intraoperative fluorescence have spread to various fields of visceral surgery. Indocyanine green (ICG)-based fluorescence angiography and the imaging systems using this certain dye are currently the cornerstone of intraoperative, fluorescence-based medical imaging. Summary: The article focuses on principles and approaches of intraoperative fluorescence in general surgery. The current clinical practice of intraoperative fluorescence and its evidence are described. Emerging new fields of application are put in a perspective. Furthermore, the technique and possible pitfalls in the performance of intraoperative ICG fluorescence angiography are described in this review article. Key Messages: Overall growing evidence suggests that intraoperative fluorescence imaging delivers valuable additional information to the surgeon, which might help to perform surgery more exactly and reduce perioperative complications. Perfusion assessment can be a helpful tool when performing critical anastomoses. There is evidence from prospective and randomized trials for the benefit of intraoperative ICG fluorescence angiography during esophageal reconstruction, colorectal surgery, and surgery for mesenteric ischemia. Most studies suggest the administration of 2.5$10 \mathrm{mg}$ of ICG. Standardized settings and documentation
\end{abstract}

are essential. The benefit of ICG fluorescence imaging for gastrointestinal sentinel node detection and detection of liver tumors and colorectal metastases of the liver cannot clearly be estimated duo to the small number of prospective studies. Critical points in the use of intraoperative fluorescence imaging remain the low standardization and reproducibility of the results and the associated difficulty in comparing the results of the existing trials. Furthermore, little is known about the influence of hemodynamic parameters on the quantitative assessment of ICG fluorescence during surgery.

(c) 2020 S. Karger AG, Basel

\section{Introduction}

Fluorescence-based imaging has evolved into an important technology in recent years. Specifically, indocyanine green (ICG)-based fluorescence imaging is now a basic tool in several fields of diagnostic and interventional medicine. Due to the physical and chemical properties of the substance, such as its rapid degradation and very rare serious adverse reactions, ICG is currently the most commonly used fluorescent agent.

ICG has found application in several fields of the general surgery, especially colorectal surgery [1], esophageal surgery [2], and emergency evaluation of intestinal perfusion in cases of mesenteric ischemia [3], kidney transplantation [4], hepatobiliary [5] and endocrine surgery [6-8]. One of the main reasons that led to the wide spread of ICG-based imaging was the launch of a series of medi- 
Table 1. Fluorescence imaging systems

\begin{tabular}{lllll}
\hline System & Producer & Light-Source & $\begin{array}{l}\text { Excitation } \\
\text { wave length }\end{array}$ & Optics \\
\hline SPY-Q & Novadaq & Laser & $805 \pm 8 \mathrm{~nm}$ & Open field \\
PinPoint & Novadaq & LED + laser & $805 \pm 8 \mathrm{~nm}$ & $0^{\circ} / 30^{\circ}$ \\
D-Light & Novadaq & Xenon & $650-800 \mathrm{~nm}$ & Open field $/ 30^{\circ}$ \\
Aim Platform & Stryker & LED + laser & $805 \mathrm{~nm}$ & $30^{\circ}$ \\
Spectrum & Quest Medical & LED + laser & $680-780 \mathrm{~nm}$ & Open field $/ 30$ \\
Fluobeam & Fluoptics & Laser & $750 \mathrm{~nm}$ & Open field \\
Visera Elite & Olympus & LED & $2 \mathrm{modes}$ & $0^{\circ} / 30^{\circ}$ \\
Viron X & Maxer Endoscopy & Not specified & $760-800 \mathrm{~nm}$ & $0^{\circ} / 30^{\circ}$ \\
\hline
\end{tabular}

cal imaging systems which revived the interest of the medical community in using intraoperative fluorescence.

This paper focuses on the current practice of ICG fluorescence in visceral surgery. Intraoperative fluorescence imaging is safe, fast, and reproducible if the surgeon is aware of the agent used, the way the agent shall be administered, and possible pit falls in the application. In visceral surgery, the most popular applications of fluorescence imaging are in the field of tissue perfusion before or after the completion of anastomoses in colorectal and esophageal surgery. Furthermore, sentinel node detection, detection of metastases, and the identification of biliary leakage are the focus of fluorescence imaging and will be discussed in this paper.

In term of the future, intraoperative fluorescent imaging might evolve and offer the surgeon even more valuable intraoperative information via new technological developments in imaging and further development of tissue or cancer specific fluorescent dyes.

\section{Fluorescence Imaging Technology}

Various fluorescence-based imaging systems are currently commercially available. The systems differ distinctively in their technology in terms of light sources, optics, and excitation [9]. Table 1 shows an overview of the different fluorescence imaging systems and their specific properties. Modern fluorescence systems have high-resolution cameras for open and laparoscopic applications. Moreover, some platforms offer fused imaging enabling real-time fluorescence imaging without having to switch off the top lights.

Recently our study group tried to clarify the advantages and disadvantages of current ICG fluorescent systems in general surgery by comparing the systems under standardized conditions ex vivo and in vivo (unpubl. data). DSouza et al. [9] did a very thorough workup of imaging systems which proved, in concordance to our data, that all systems detect ICG fluorescence and are of clinical usability. Upon closer inspection, however, differences in the quality of images, noise ratio and image fusion seem to become visually evident. In ICG fluorescence angiography, LED systems tend to need higher dosages of ICG compared to current laser-based imaging systems. Moreover, most of the systems do not offer a quantification tool yet. Until now it is unknown how far these systems deliver comparable results between each other in the estimation of, e.g., tissue perfusion. Autoadjustment software within some of the systems might even hamper the clinical comparability of studies and applications between different systems [9].

In the near future, companies might offer simultaneous fluorescent imaging using several different dyes even under with the theater lights on [10]. This fact would allow the simultaneous coloring - and therefore detection - of different structures, like nerves, tumors, and vessels. In fact, since the description of the green fluorescent protein by the former Nobel prize winner Roger Tsien, various specific fluorescent dyes have been developed [11]. Nerve-specific and tissue- or tumor-specific dyes are available for experimental settings, lacking approval for clinical use today [12]. Ultimately mentioned, studies examining the binding of fluorophores (like ICG) to antibodies are opening new horizons in the clinical use of these dyes in medical oncology $[13,14]$.

\section{Indocyanine Green}

ICG was used as a photographic dye during the Second World War and it was first implemented in medical imaging in cardiac and liver function tests $[15,16]$. Severe allergic reactions associated with the use of ICG are very rare with an incidence of $0.05 \%$ and mostly occur in patients allergic to iodine. Furthermore, potential cross reactions with penicillin have been suggested.

One of the main issues in the clinical (and experimental) use of ICG is the inconsistence of the available data with regard to the physical and chemical properties of 
ICG itself. In most trials ICG is diluted in aqua and administered intravenously. However, information like the dosage, the way of application (bolus or fractionated), and the time from when the ICG bottle was opened (ICG degrades over time) are usually not mentioned in most of the available studies.

For intravenous use the half-life of ICG is about 2.4 min. The substance is then metabolized by the liver and excreted in the bile, suggesting an enhanced fluorescence effect in patients with impaired liver function [17].

In a previous publication of our group [18], the ICG properties were studied. With regards to the stability of the substance when dissolved in aqua and blood the available literature was widely unprecise and the was ranging from complete degradation within 20 min [19] to stabil-

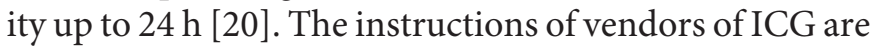
also different, ranging from immediate use of the reconstituted ICG solution (Diagnostic Green, Belgium) to usage within 6 h (Akorn, USA) [18]. Furthermore, we tested the influence of day light and temperature on the degradation of ICG. When diluted in water and stored at $4{ }^{\circ} \mathrm{C}$ in the dark, ICG was stable for 3 days, losing as few as $20 \%$ of fluorescence intensity within this time span. When ICG was incubated at $37^{\circ} \mathrm{C}$ in whole blood under light exposure, it only stayed stable for $5 \mathrm{~h}$. Based on our experiments the durability of the rather expensive dye was longer than expected. Contrary to the manufacturer's recommendation, we suggest that ICG should be used within 1 or 2 days after resolution if the ICG solution is stored at $4{ }^{\circ} \mathrm{C}[18]$ and aseptic conditions are warranted.

\section{Tissue Angiography: Approach and Possible Pit Falls}

When implementing ICG fluorescence to assess tissue perfusion, the anesthetist administers ICG when requested by the surgeon followed by an immediate intravenous flush of $10 \mathrm{~mL}$ of an isotonic solution. The team must be trained, as the time point of administration, the start of the imaging process, and detection of the signal in the area of interest must be coordinated (Fig. 1). Uncoordinated administration or image capturing long after/or even before injecting the dye might lead to acquisition of false data causing misinterpretation of the tissue perfusion. To assess intestinal perfusion most studies suggest 2.5-10 mg of ICG depending on the body weight and the imaging system used. As mentioned above, LED and filter systems might need higher dosages compared to laser systems. The time to detect a fluorescence signal varies between 25 and $60 \mathrm{~s}$ after administration. The signal peaks around 30-45 s after administration (end of arterial phase) and flattens thereafter by losing $40 \%$ of its peak intensity within 1-2 min (venous wash out phase; Fig. 1). Peak and washout strongly depend on the

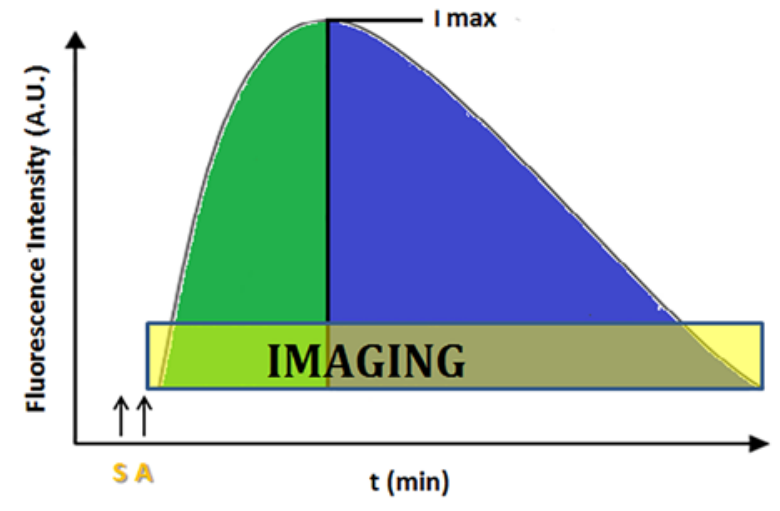

Fig. 1. ICG fluorescence angiography. X-axis: time after ICG injection. Y-axis: fluorescence intensity in AU;Imax: peak intensity of the fluorescence signal. Arterial inflow is marked green, and venous drainage is marked blue. Clear communication between the surgeon $(\mathrm{S})$ and the anesthesiologist $(\mathrm{A})$ is necessary for capturing the full ingress and egress phase of the fluorescence signal in a video sequence by the imaging system. Modified after Rother et al. [4].

patient's circulatory condition and on possible cardiocirculatory support such as inotropes. High doses of inotropes might strongly influence the intensity of tissue perfusion and might decrease the intensity of fluorescence.

It is common sense that ICG applications should be documented following certain standards, i.e., documentation of the time point, the precise dose of ICG administered, the number of administrations, the time to the first appearance of a fluorescence signal, and the side effects of the dye. The correlation of clinical and visual evaluation using fluorescence angiography should be documented in each case. If the surgical approach is altered due to fluorescence findings, it should be noted as well.

Standardized settings and documentation are essential, as they rule out most of the possible pitfalls in ICG fluorescence angiography. ICG has a nonlinear quantum yield compared to the concentration [21]. Its dosage affects light absorption and, in many cases, less than the manufacturer's suggested dosage is sufficient when using modern fluorescence imaging systems [4]. The detection of a weak signal can be due to degradation by light or heat over the time as mentioned above [18]. Another reason for weak signals might be a long distance between the camera and the tissue, a too slow injection of the dye, or a lack of flushing afterwards. Another point is ambient light for some systems or theatre lights, as that might act as a scatterer. In contrast, a strong signal or blooming effect might be due to too high doses, miscommunication, and light scattering on the tissue in open surgery. 


\section{Evidence in Colorectal Surgery}

The largest amount of studies of ICG perfusion is found in the field of colorectal surgery and performance of anastomoses. Several systematic reviews collected and analyzed data in the field of colorectal anastomoses with similar results and conclusions [22, 23]. Degett et al. [22] included 17 studies and reported a significantly lower leakage rate with ICG angiography (3.8\%) compared to surgery without intraoperative fluorescent imaging (7.3\%). The amount of high-quality prospective randomized trials in this field is scarce, however. Jafari et al. [24] have reported a change of surgical plan in $8 \%$ of cases and a leakage rate of only $2 \%$ after using ICG angiography in the prospective randomized PILLAR II trial addressing high and low anterior resections. In the latest meta-analysis on ICG fluorescence in colorectal surgery, $1,302 \mathrm{pa}-$ tients recruited in 5 nonrandomized trials were shown to have a significant reduction in anastomotic leaks in colorectal cancer surgery and rectal cancer surgery [23]. The latest and largest European prospective multicenter trial included 504 patients $(65 \%$ colorectal cancer patients) from 3 colorectal centers and reported a feasibility of $100 \%$ with an additional operating time of $4 \mathrm{~min}$. ICG led to a change in the operative strategy in $6 \%$ of the cases [25]. A prospective European randomized multicenter trial (IntAct) has just been started evaluating the influence of intraoperative ICG fluorescence on postoperative leakage in deep anterior resections for colorectal cancer [26].

\section{Evidence in Emergency Surgery and Mesenteric Ischemia}

Acute mesenteric ischemia remains associated with a high morbidity and a poor outcome [27]. Evaluation of bowel vitality is still subjective, mostly based on surgical experience and can lead to resection of segments that could potentially recover. ICG fluorescence angiography could be an objective, noninvasive method to assess intestinal viability to determine the extent of ischemia correctly. In a porcine study the real-time assessment of intestinal viability by ICG fluorescence intensity was quantitatively assessed by calculating the time to peak in ischemic and nonischemic intestinal segments [28]. In another experimental study assessing the intestinal perfusion, the group of Diana et al. [28] concluded that a reduced perfusion of $25 \%$ certainly impairs anastomotic healing without generating surgical complications.

Numerous feasibility reports and small case series have been reported on mesenteric malperfusion and the application of ICG fluorescence for the evaluation of intestinal vitality. In a series of 54 patients, we could show that intraoperative ICG angiography influences surgical decision making in $30 \%$ of cases [3]. ICG fluorescence was found to be superior to visual assessment alone and has an impact on surgical decision making, especially with regard to the decision of whether to perform secondlook operations, resection, or even revascularization. Comparable findings were published by the Geneva group simultaneously [29].

Hyperintensity due to capillary leakage and consecutive pooling of ICG in the tissue may lead to misinterpretation. In the case of visual assessment alone, there is a risk of assessing previously damaged areas as adequately perfused because of the ongoing fluorescence within the tissue. Normally, this pooling fluorescence lasts longer due to the loss of epithelial integrity. A well-perfused tissue shows a quick inflow of ICG and a slower, but visible, outflow (Fig. 1). Due to liver failure and sepsis, ICG might also persist much longer within the circulation so that remaining fluorescence in the liver and bowel might impair interpretation of repeated ICG perfusion assessments during second look laparotomies.

Furthermore, hemodynamic alterations during the operation or during the patient's course might lead to the detection of differing ICG fluorescence intensities. Hemodynamics, such as cardiac output, blood pressure, vascular resistance, volume load, and catecholamine therapy, can influence the results. So far, these important confounding factors seem to be underestimated in the literature. It is well-known that liver function and alterations of cardiac output affect the plasma disappearance of ICG [30-32]. This might influence the washout phase of the ICG signal. However, further studies are needed to determine the influence of hemodynamic parameters on the ingress and egress of the ICG signal in quantitative assessment of ICG fluorescence during surgery. In the clinical setting, those parameters must be taken into consideration for valid measurements. If a stable setting is not possible, either a ratio to a baseline value or comparison to a nonischemic area during the same measurement is mandatory. For the purpose of a valid quantitative assessment of ICG fluorescence angiography the "background-subtracted peak fluorescence intensity" and the "slope of fluorescence intensity" have been described [33, 34].

\section{Evidence in Esophageal Reconstruction}

ICG tissue angiography has been used early to assess graft perfusion before esophageal reconstruction in various studies. The published literature can be divided into studies evaluating ICG perfusion to minimize leakage rates after placing the anastomosis and studies to describe the root of gastric perfusion without evaluation of clinical 
consequences. Zehetner et al. [35] concluded in their study in 150 patients undergoing esophagectomy that intraoperative real-time assessment of perfusion correlated with the likelihood of an anastomotic leak and confirmed the critical relationship between adequate perfusion and anastomotic healing [35]. In our own series, in a nonrandomized prospective setting we could confirm these results [2]. Two recent systematic reviews and meta-analyses have just reported on the use of intraoperative ICG fluorescence in esophageal surgery $[36,37]$. Both reviews offer encouraging evidence for the use of ICG in gastric conduit reconstruction as evidenced by a $69 \%$ reduction in anastomotic leaks when combined with intraoperative interventions. However, poor data quality and heterogeneity in reported variables limit the validity of these findings. Tools for quantitative assessment of perfusion are available and can provide an objective and standardized approach to evaluate the effectiveness of ICG [34]. Future studies should incorporate these measures in randomized controlled trials to generate more robust evidence in this area.

\section{ICG Fluorescence of the Bile and Liver Masses}

After systemic injection of ICG, an increase in the detection rates of colorectal liver metastases and hepatocellular carcinomas has been reported in several series. However, sensitivity and specificity differ significantly [38-42]. In a nutshell, ICG is administered at a minimum dose of $10 \mathrm{mg}$ (we recommend 25-50 mg) 24-72 h prior to surgery to enable metabolization in the liver and accumulation in the bile. In a study for common bile duct identification in cholecystectomies the dosage and administration interval ( $24 \mathrm{~h}$ compared to $30 \mathrm{~min}$ ) of ICG before surgery have been shown to alter the contrast of the bile duct in relation to background normal liver tissue [43]. Intraoperative ICG fluorescence for the prevention of bile duct injury during cholecystectomies has been reported in numerous publications. van de Graaf et al. [44] reported a median detection rate of $76 \%$ for the common bile duct, $88 \%$ for the cystic duct and common hepatic duct confluence, and $100 \%$ for the cystic duct. A total of 863 patients were included in 16 studies. No intraoperative bile duct injury occurred when using ICG fluorescence. Regarding sample sizes and study designs, the authors concluded that there is currently no preferred method to prevent bile duct injury in cholecystectomies [44].

ICG is metabolized in the liver and accumulates in regions of delayed bile metabolism, as found in primary liver tumors and colorectal metastases [38, 41]. The tumor can be visualized by ICG fluorescence at a size of just 200 tumor cells, enabling surgeons to visualize foci of a mini- mum of $1 \mathrm{~mm}$ [42]. A major limitation in the visualization of liver masses with the available fluorescence systems is the maximum depth of infiltration of up to 10 $\mathrm{mm}$. A combination of application of intraoperative sonography and fluorescence was shown to increase the detection rate of colorectal metastases [39-41, 45]. In patients with liver fibrosis, however, regions with delayed bile metabolism might give false-positive fluorescence.

ICG fluorescence molecular imaging technology can be used to qualitatively and quantitatively analyze pathological changes at a molecular and cellular level in vivo, to objectively display liver tumor boundary information, to define liver tumor boundaries, and to detect residual lesions, achieving an intraoperative real-time staining and navigation of the liver parenchyma in the target area $[38,46,47]$. Most of the studies have been conducted as case series or case reports. The latest meta-analysis included studies on 587 patients showing that ICG fluorescence in the field of liver surgery does decrease operative time, blood loss, hospital stay, and postoperative complications [5]. Bearing in mind the heterogeneity and quality of the current data available, there is need for future prospective randomized trials.

\section{ICG Fluorescence for Sentinel Node Detection}

This is another important topic in several fields of surgery, urology, gynecology, and general surgery [48-50]. ICG has been shown to be comparable to blue dye and radioactive markers in several studies for breast cancer. The future will tell whether it is safe to solely rely on ICG as a dye. However, the latest meta-analysis showed a significantly better sentinel node detection by ICG compared to the standard radioisotope method $[48,51]$. The prospectively randomized FILM trial evaluated ICG to be superior in lymph node detection compared to isosulfan blue dye in patients with stage I endometrial or cervical cancer [52]. In the meanwhile, the design and results of the study provided groundwork for the FDA licensing of ICG for lymph node mapping. NCCN guidelines mention sentinel lymph node mapping by ICG in cervical cancer patients [53].

For colorectal cancer, several feasibility studies have been carried out without showing any clinical consequence or resulting in a prospective randomized trial so far. For colorectal cancer but also for lower rectal tumors sentinel node imaging is feasible, especially to detect the lymphatic drainage across the lateral lymph nodes [5458]. In esophageal and gastric cancer, ICG has been shown to work as a feasible tracer for regional lymphatic mapping [59, 60]. Studies in sentinel node detection in all gastrointestinal cancer fields are phase I/II trials with a wide variation in the number, dosage, and location of dye 
injection (subserosal vs. submucosal) and the time between injection and sentinel node mapping [61]. Practically, ICG solutions between $0.5 \mathrm{mg}$ and $5 \mathrm{mg} / \mathrm{mL}$ are injected proximally and distally of the tumor ( 2 times 1 $\mathrm{mL}$ ) or around the tumor (4 times $0.5 \mathrm{~mL}$ ).

Although current data favoring intraoperative detection of lymph node metastasis and peritoneal metastasis with ICG in colorectal cancer is scarce, ICG fluorescence could potentially improve the staging technique and treatment of patients especially in the context of molecular imaging when conjugated to cancer related antibodies [62].

\section{Conclusion}

This review presents an overview of the current technological concepts and principles of ICG fluorescence imaging as well as intraoperative imaging platforms available. The ICG tissue angiography concept described in this article provides the surgeon with a clear advice on how to perform intraoperative ICG fluorescence angiography pointing out possible pitfalls. Although there is emerging evidence of the usefulness of intraoperative ICG angiography in esophageal reconstruction, colorectal surgery, and mesenteric ischemia, the amount of highquality studies is still scarce. The great amount of case series and the small amount of prospective randomized trials results in a high data heterogeneity reducing the reproducibility and generalizability of the results.

The evidence of a potential benefit associated with the ICG fluorescence imaging for gastrointestinal sentinel node detection and detection of liver tumors and colorectal metastases of the liver is also limited.

Overall, in just a few years' time, the intraoperative fluorescence imaging with ICG has found numerous interesting applications in visceral surgery raising the hope that future studies will allow us to perform more precise surgery with less perioperative complications.

\section{Disclosure Statement}

Kai Nowak works as a consultant for Stryker and Novadaq in the field of intraoperative fluorescence imaging.

\section{Funding Sources}

Not applicable.

\section{Author Contributions}

Kai Nowak and Andreas Lutz Heinrich Gerken wrote and contributed to this paper equally. Ioannis Karampinis participated in revision and adaptation of this paper.

\section{References}

1 Boni L, Fingerhut A, Marzorati A, Rausei S, Dionigi G, Cassinotti E. Indocyanine green fluorescence angiography during laparoscopic low anterior resection: results of a casematched study. Surg Endosc. 2017 Apr;31(4): 1836-40.

2 Karampinis I, Ronellenfitsch U, Mertens C, Gerken A, Hetjens S, Post S, et al. Indocyanine green tissue angiography affects anastomotic leakage after esophagectomy: a retrospective, case-control study. Int J Surg. 2017 Dec;48: 210-14.

3 Karampinis I, Keese M, Jakob J, Stasiunaitis V, Gerken A, Attenberger U, et al. Indocyanine Green Tissue Angiography Can Reduce Extended Bowel Resections in Acute Mesenteric Ischemia. J Gastrointest Surg. 2018 Dec; 22(12):2117-24.

4 Rother U, Gerken ALH, Karampinis I, Klumpp M, Regus S, Meyer A, et al. Dosing of indocyanine green for intraoperative laser fluorescence angiography in kidney transplantation. Microcirculation. 2017 Nov; 24(8).

5 Qi C, Zhang H, Chen Y, Su S, Wang X, Huang $\mathrm{X}$, et al. Effectiveness and safety of indocyanine green fluorescence imaging-guided hepatectomy for liver tumors: a systematic review and first meta-analysis. Photodiagn Photodyn Ther. 2019 Dec;28:346-53.
6 Karampinis I, Di Meo G, Gerken A, Stasiunaitis V, Lammert A, Nowak K. [Intraoperative Indocyanine Green Fluorescence to Assure Vital Parathyroids in Thyroid Resections]. Zentralbl Chir. 2018 Aug;143(4):380-4.

7 Vidal Fortuny J, Sadowski SM, Belfontali V, Guigard S, Poncet A, Ris F, et al. Randomized clinical trial of intraoperative parathyroid gland angiography with indocyanine green fluorescence predicting parathyroid function after thyroid surgery. Br J Surg. 2018 Mar; 105(4):350-7.

8 Di Meo G, Karampinis I, Gerken A, Lammert A, Pellicani S, Nowak K. Indocyanine green fluorescence angiography can guide intraoperative localization during parathyroid surgery. Scand J Surg. 2019 Sep: 1457496919877581.

9 DSouza AV, Lin H, Henderson ER, Samkoe KS, Pogue BW; AV DS. Review of fluorescence guided surgery systems: identification of key performance capabilities beyond indocyanine green imaging. J Biomed Opt. 2016 Aug;21(8):80901.

10 Dimitriadis N, Grychtol B, Maertins L, Behr T, Themelis G, Deliolanis NC. Simultaneous real-time multicomponent fluorescence and reflectance imaging method for fluorescenceguided surgery. Opt Lett. 2016 Mar;41(6): 1173-6.
11 Tsien RY. Constructing and exploiting the fluorescent protein paintbox (Nobel Lecture). Angew Chem Int Ed Engl. 2009;48(31):5612-26.

12 Whitney MA, Crisp JL, Nguyen LT, Friedman B, Gross LA, Steinbach P, et al. Fluorescent peptides highlight peripheral nerves during surgery in mice. Nat Biotechnol. 2011 Apr; 29(4):352-6.

13 Ogawa M, Kosaka N, Choyke PL, Kobayashi $H$. In vivo molecular imaging of cancer with a quenching near-infrared fluorescent probe using conjugates of monoclonal antibodies and indocyanine green. Cancer Res. 2009 Feb; 69(4):1268-72.

14 Anayama T, Qiu J, Chan H, Nakajima T, Weersink R, Daly M, et al. Localization of pulmonary nodules using navigation bronchoscope and a near-infrared fluorescence thoracoscope. Ann Thorac Surg. 2015 Jan;99(1): 224-30.

15 Fox IJ, Brooker LG, Heseltine DW, Essex HE, Wood EH. A tricarbocyanine dye for continuous recording of dilution curves in whole blood independent of variations in blood oxygen saturation. Proc Staff Meet Mayo Clin. 1957 Sep;32(18):478-84.

16 Alander JT, Kaartinen I, Laakso A, Pätilä T, Spillmann T, Tuchin VV, et al. A review of indocyanine green fluorescent imaging in surgery. Int J Biomed Imaging. 2012;2012:940585. 
17 Ott P. Hepatic elimination of indocyanine green with special reference to distribution kinetics and the influence of plasma protein binding. Pharmacol Toxicol. 1998;83 Suppl 2: $1-48$.

18 Mindt S, Karampinis I, John M, Neumaier M, Nowak K. Stability and degradation of indocyanine green in plasma, aqueous solution and whole blood. Photochem Photobiol Sci. 2018 Sep;17(9):1189-96.

19 Hollins B, Noe B, Henderson JM. Fluorometric determination of indocyanine green in plasma. Clin Chem. 1987 Jun;33(6):765-8.

20 Gathje J, Steuer RR, Nicholes KR. Stability studies on indocyanine green dye. J Appl Physiol. 1970 Aug;29(2):181-5.

21 Benson RC, Kues HA. Fluorescence properties of indocyanine green as related to angiography. Phys Med Biol. 1978 Jan;23(1):159-63

22 Degett TH, Andersen HS, Gögenur I. Indocyanine green fluorescence angiography for intraoperative assessment of gastrointestinal anastomotic perfusion: a systematic review of clinical trials. Langenbecks Arch Surg. 2016 Sep;401(6):767-75.

23 Blanco-Colino R, Espin-Basany E. Intraoperative use of ICG fluorescence imaging to reduce the risk of anastomotic leakage in colorectal surgery: a systematic review and meta-analysis. Tech Coloproctol. 2018 Jan; 22(1):15-23.

24 Jafari MD, Wexner SD, Martz JE, McLemore EC, Margolin DA, Sherwinter DA, et al. Perfusion assessment in laparoscopic left-sided/ anterior resection (PILLAR II): a multi-institutional study. J Am Coll Surg. 2015 Jan; 220(1):82-92.e1.

25 Ris F, Liot E, Buchs NC, Kraus R, Ismael G, Belfontali V, et al.; Near-Infrared Anastomotic Perfusion Assessment Network VOIR. Multicentre phase II trial of near-infrared imaging in elective colorectal surgery. Br J Surg. 2018 Sep;105(10):1359-67.

26 Armstrong G, Croft J, Corrigan N, Brown JM, Goh V, Quirke P, et al. IntAct: intra-operative fluorescence angiography to prevent anastomotic leak in rectal cancer surgery: a randomized controlled trial. Colorectal Dis. 2018 Aug;20(8):O226-34.

27 Meng X, Liu L, Jiang H. Indications and procedures for second-look surgery in acute mesenteric ischemia. Surg Today. 2010 Aug; 40(8):700-5.

28 Diana M, Halvax P, Dallemagne B, Nagao Y, Diemunsch P, Charles AL, et al. Real-time navigation by fluorescence-based enhanced reality for precise estimation of future anastomotic site in digestive surgery. Surg Endosc. 2014 Nov;28(11):3108-18.

29 Liot E, Assalino M, Buchs NC, Schiltz B, Douissard J, Morel P, et al. Does near-infrared (NIR) fluorescence angiography modify operative strategy during emergency procedures? Surg Endosc. 2018 Oct;32(10):4351-6.

30 Hofmann D, Thuemer O, Schelenz C, van Hout N, Sakka SG. Increasing cardiac output by fluid loading: effects on indocyanine green plasma disappearance rate and splanchnic microcirculation. Acta Anaesthesiol Scand. 2005 Oct;49(9):1280-6.
31 Janssen MW, Druckrey-Fiskaaen KT, Omidi L, Sliwinski G, Thiele C, Donaubauer B, et al. Indocyanine green $\mathrm{R} 15$ ratio depends directly on liver perfusion flow rate. J Hepatobiliary Pancreat Sci. 2010 Mar;17(2):180-5.

32 Sakka SG. Assessment of liver perfusion and function by indocyanine green in the perioperative setting and in critically ill patients. J Clin Monit Comput. 2018 Oct;32(5):787-96.

33 Detter C, Wipper S, Russ D, Iffland A, Burdorf L, Thein E, et al. Fluorescent cardiac imaging: a novel intraoperative method for quantitative assessment of myocardial perfusion during graded coronary artery stenosis. Circulation. 2007 Aug;116(9):1007-14.

34 Dupree A, Riess H, Detter C, Debus ES, Wipper SH. Utilization of indocynanine green fluorescent imaging (ICG-FI) for the assessment of microperfusion in vascular medicine. Innov Surg Sci. 2018 Sep;3(3):193-201.

35 Zehetner J, DeMeester SR, Alicuben ET, Oh DS, Lipham JC, Hagen JA, et al. Intraoperative Assessment of Perfusion of the Gastric Graft and Correlation With Anastomotic Leaks After Esophagectomy. Ann Surg. 2015 Jul;262(1):74-8.

36 Ladak F, Dang JT, Switzer N, Mocanu V, Tian C, Birch D, et al. Indocyanine green for the prevention of anastomotic leaks following esophagectomy: a meta-analysis. Surg Endosc. 2019 Feb;33(2):384-94.

37 Slooter MD, Eshuis WJ, Cuesta MA, Gisbertz SS, van Berge Henegouwen MI. Fluorescent imaging using indocyanine green during esophagectomy to prevent surgical morbidity: a systematic review and meta-analysis. J Thorac Dis. 2019 Apr;11(S5 Suppl 5):S75565.

38 Ishizawa T, Fukushima N, Shibahara J, Masuda K, Tamura S, Aoki T, et al. Real-time identification of liver cancers by using indocyanine green fluorescent imaging. Cancer. 2009 Jun;115(11):2491-504.

39 Uchiyama K, Ueno M, Ozawa S, Kiriyama S, Shigekawa Y, Yamaue H. Combined use of contrast-enhanced intraoperative ultrasonography and a fluorescence navigation system for identifying hepatic metastases. World J Surg. 2010 Dec;34(12):2953-9.

40 Peloso A, Franchi E, Canepa MC, Barbieri L, Briani L, Ferrario J, et al. Combined use of intraoperative ultrasound and indocyanine green fluorescence imaging to detect liver metastases from colorectal cancer. $\mathrm{HPB}\left(\mathrm{Ox}^{-}\right.$ ford). 2013 Dec;15(12):928-34.

41 van der Vorst JR, Schaafsma BE, Hutteman M, Verbeek FP, Liefers GJ, Hartgrink HH, et al. Near-infrared fluorescence-guided resection of colorectal liver metastases. Cancer. 2013 Sep;119(18):3411-8.

42 Ishizawa T, Masuda K, Urano Y, Kawaguchi Y, Satou S, Kaneko J, et al. Mechanistic background and clinical applications of indocyanine green fluorescence imaging of hepatocellular carcinoma. Ann Surg Oncol. 2014 Feb; 21(2):440-8

43 Verbeek FP, Schaafsma BE, Tummers QR, van der Vorst JR, van der Made WJ, Baeten $\mathrm{CI}$, et al. Optimization of near-infrared fluorescence cholangiography for open and laparoscopic surgery. Surg Endosc. 2014 Apr; 28(4):1076-82.
44 van de Graaf FW, Zaimi I, Stassen LPS, Lange JF. Safe laparoscopic cholecystectomy: a systematic review of bile duct injury prevention. Int J Surg. 2018 Dec;60:164-72.

45 Kaibori M, Matsui K, Ishizaki M, Iida H, Okumura T, Sakaguchi T, et al. Intraoperative Detection of Superficial Liver Tumors by Fluorescence Imaging Using Indocyanine Green and 5-aminolevulinic Acid. Anticancer Res. 2016 Apr;36(4):1841-9.

46 Inoue Y, Arita J, Sakamoto T, Ono Y, Takahashi M, Takahashi Y, et al. Anatomical Liver Resections Guided by 3-Dimensional Parenchymal Staining Using Fusion Indocyanine Green Fluorescence Imaging. Ann Surg. 2015 Jul;262(1):105-11.

47 Zhang YM, Shi R, Hou JC, Liu ZR, Cui ZL, Li $\mathrm{Y}$, et al. Liver tumor boundaries identified intraoperatively using real-time indocyanine green fluorescence imaging. J Cancer Res Clin Oncol. 2017 Jan;143(1):51-8.

48 Benson J. Indocyanine Green Fluorescence for Sentinel Lymph Node Detection in Early Breast Cancer. Ann Surg Oncol. 2016 Jan; 23(1):6-8.

49 Emile SH, Elfeki H, Shalaby M, Sakr A, Sileri $\mathrm{P}$, Laurberg S, et al. Sensitivity and specificity of indocyanine green near-infrared fluorescence imaging in detection of metastatic lymph nodes in colorectal cancer: systematic review and meta-analysis. J Surg Oncol. 2017 Nov;116(6):730-40.

50 Aoun F, Albisinni S, Zanaty M, Hassan T, Janetschek G, van Velthoven R. Indocyanine green fluorescence-guided sentinel lymph node identification in urologic cancers: a systematic review and meta-analysis. Minerva Urol Nefrol. 2018 Aug;70(4):361-9.

51 Sugie T, Ikeda T, Kawaguchi A, Shimizu A, Toi M. Sentinel lymph node biopsy using indocyanine green fluorescence in early-stage breast cancer: a meta-analysis. Int J Clin Oncol. 2017 Feb;22(1):11-7.

52 Frumovitz M, Plante M, Lee PS, Sandadi S, Lilja JF, Escobar PF, et al. Near-infrared fluorescence for detection of sentinel lymph nodes in women with cervical and uterine cancers (FILM): a randomised, phase 3, multicentre, non-inferiority trial. Lancet Oncol. 2018 Oct;19(10):1394-403.

53 Koh WJ, Abu-Rustum NR, Bean S, Bradley K, Campos SM, Cho KR, et al. Cervical Cancer, Version 3.2019, NCCN Clinical Practice Guidelines in Oncology. J Natl Compr Canc Netw. 2019 Jan;17(1):64-84.

54 Nagata K, Endo S, Hidaka E, Tanaka J, Kudo SE, Shiokawa A. Laparoscopic sentinel node mapping for colorectal cancer using infrared ray laparoscopy. Anticancer Res. 2006 MayJun;26 3B:2307-11.

55 Kawahara H, Nimura H, Watanabe K, Kobayashi T, Kashiwagi H, Yanaga K. Where does the first lateral pelvic lymph node receive drainage from? Dig Surg. 2007;24(6):413-7.

56 Cahill RA, Anderson M, Wang LM, Lindsey I, Cunningham C, Mortensen NJ. Near-infrared (NIR) laparoscopy for intraoperative lymphatic road-mapping and sentinel node identification during definitive surgical resection of early-stage colorectal neoplasia. Surg Endosc. 2012 Jan;26(1):197-204. 
57 Liberale G, Galdon MG, Moreau M, Vankerckhove S, El Nakadi I, Larsimont D, et al. Ex vivo detection of tumoral lymph nodes of colorectal origin with fluorescence imaging after intraoperative intravenous injection of indocyanine green. J Surg Oncol. 2016 Sep; 114(3):348-53.

58 Zhou SC, Tian YT, Wang XW, Zhao CD, Ma $\mathrm{S}$, Jiang J, et al. Application of indocyanine green-enhanced near-infrared fluorescenceguided imaging in laparoscopic lateral pelvic lymph node dissection for middle-low rectal cancer. World J Gastroenterol. 2019 Aug; 25(31):4502-11.
59 Hachey KJ, Gilmore DM, Armstrong KW, Harris SE, Hornick JL, Colson YL, et al. Safety and feasibility of near-infrared image-guided lymphatic mapping of regional lymph nodes in esophageal cancer. J Thorac Cardiovasc Surg. 2016 Aug; 152(2):546-54.

60 He M, Jiang Z, Wang C, Hao Z, An J, Shen J. Diagnostic value of near-infrared or fluorescent indocyanine green guided sentinel lymph node mapping in gastric cancer: A systematic review and meta-analysis. J Surg Oncol. 2018 Dec;118(8):1243-56.
61 Ankersmit M, Bonjer HJ, Hannink G, Schoonmade LJ, van der Pas MH, Meijerink WJ. Near-infrared fluorescence imaging for sentinel lymph node identification in colon cancer: a prospective single-center study and systematic review with meta-analysis. Tech Coloproctol. 2019 Dec;23(12):1113-26.

62 Boogerd LS, Hoogstins CE, Schaap DP, Kusters M, Handgraaf HJ, van der Valk MJ, et al. Safety and effectiveness of SGM-101, a fluorescent antibody targeting carcinoembryonic antigen, for intraoperative detection of colorectal cancer: a dose-escalation pilot study. Lancet Gastroenterol Hepatol. 2018 Mar;3(3):181-91. 